\title{
Back to Basics: Limitations of Research Influencing the Human Brain Project
}

\section{Ewing GW*}

Mimex Montague Healthcare Limited, Cotgrave, Nottinghamshire, England, UK

\begin{abstract}
In an effort to unravel the workings of the human brain the European Commission established the Human Brain Project (2013) however it has been subject to intense rescrutiny, criticism and political infighting. Accordingly the aim of this article is to provide a critical review of whether, at least from the technical perspective, such criticisms are justified.

The author is in the privileged position of being able to do so because he heads a company which is in the unique position of commercialising a technology, developed by its Technical Director Dr. Igor Grakov (launched in its first commercial version in 1999), which is based upon a precise, sophisticated, and detailed mathematical model of the autonomic nervous system i.e. that cognitive input can be used as the data sets for a neural simulation technique and/or mathematical model which links brain function to the regulated function of the body's physiological and/or functional systems, the organs which are essential components of these systems, and of the pathological changes to cellular and molecular biology which are the consequence of systemic dysfunction. In other words the Strannik technology developed by Dr. Grakov meets several of the key aims and objectives of the Human Brain Project.

This article highlights (i) fundamental limitations of current diagnostic methods which will severely constrain the ability of researchers to reach a successful conclusion; (ii) fundamental limitations of medical research which ignore basic principles of chemistry and widely recognised (but unfashionable) phenomena; (iii) the assumption that there is a healthy and/or 'unhealthy' brain although clearly the health of the brain is influenced by stress, nutritional deficits and emergent pathologies; (iv) it questions the need for 'big data' rather than investment in the basic research to identify the fundamental scientific principles; and $(v)$ it is critical of the way in which contemporary biomedical research overlooks the complex and wholistic way in which the body functions.
\end{abstract}

Keywords: Human brain project; Strannik; Autonomic nervous system; Virtual scanning; Mathematical model

\section{Introduction}

The Human Brain Project has been dogged by personal infighting. An editorial in Nature (dated $24^{\text {th }}$ March 2015) the authors discussed how an independent report into this controversial project has encountered problems of governance and of scientific direction [1]. The report stated that it is premature to simulate the function of the whole-brain, that the HBP should - like the Brain Initiative - refocus on enabling methods and technologies i.e. software and hardware platforms for neuroinformatics, that such technology platforms should be developed and validated by interdisciplinary collaborations involving cognitive and systems neuroscientists; and that they should address problems e.g., goal-directed decision-making.

In an extraordinary twist we are able to comment upon a technology, Strannik, which already meets several of the key aims and objectives of the Human Brain Project [2]. The existence of this technology enables us to comment upon fundamental conceptual limitations upon which the Human Brain Project was conceived and also upon comments made in the independent report.

Such a technology is possible - it already exists and is fully developed - and meets several of the key aims and objectives of the Human Brain Project. It is a cognitive technology which embodies many of the approaches which have been adopted by cognitive neuroscientists thereby satisfying the concerns of the independent reviewer and of a subsequent $\mathrm{CEOI}$ for greater input by cognitive researchers. Furthermore the existence of such a technology is immensely significant e.g., if such a technology has been developed, which is based upon a precise understanding of how the brain regulates the autonomic nervous system, there will be less need for what has become 'big data' or in 'informatics' to decipher how the body functions and/or the relationship between the genes [3]; vastly reduce the cost and complexity of medical diagnosis; move to a preventative healthcare model; improve medical outcomes by augmenting drug-treatment protocols with non-drug therapeutic programmes; reduce the flow of patients through primary, secondary and tertiary care; improve quality of life; etc. In order to explain the essential principles upon which Strannik technology is based we have to address fundamental limitations of contemporary biomedical research.

\section{Background}

In chemistry we are taught the fundamental basics of chemistry e.g., how a chemical will react with another chemical and how this reaction is governed by its reaction kinetics i.e. the factors which influence the rate of this reaction and how this influences the structure, quality and/or quantity of metabolites. In the study of 'how one chemical will react with another chemical and how this reaction is governed by its reaction kinetics' the level of a particular chemical cannot therefore be

*Corresponding author: Ewing GW, Mimex Montague Healthcare Limited, Cotgrave, Nottinghamshire, England, UK, Tel: 0044 7885755847; Fax: 0044 1159899826; E-mail: graham.ewing@mmhcl.co.uk

Received October 01, 2015; Accepted October 20, 2015; Published October 22 2015

Citation: Ewing GW (2015) Back to Basics: Limitations of Research Influencing the Human Brain Project. J Comput Sci Syst Biol 8:6 322-326. doi:10.4172/jcsb.1000206

Copyright: (c) 2015 Ewing GW. This is an open-access article distributed under the terms of the Creative Commons Attribution License, which permits unrestricted use, distribution, and reproduction in any medium, provided the original author and source are credited. 
an accurate determinant. To illustrate the point: (i) there can be a high level of a reactant and a low rate of reaction or there can be a low level of a reactant and a high rate of reaction; (ii) the reaction conditions may not be able to sustain the reaction or may alter the metabolic outcomes; (iii) If measuring a metabolite the rate of reaction and of the complex factors which affect the rate of reaction will influence 'the spectrum of metabolites' which are produced and not just a single metabolite e.g., the ratio of glycated proteins which are diabetic metabolites; (iv) it is assumed that a single biomarker can be used as an accurate determinant yet the body does not conform to reductionist ideals. It is a neurally regulated, multi-systemic, entity in which many changes of molecular biology are the consequence of this process, not its cause!

If you develop a back complaint as a result of stress the problem is the stress. The back complaint is the consequence of the stress. If you develop diabetes/obesity as a result of eating too much or too little exercise the problem is that you eat too much and/or have too little exercise and that there is an imbalance between/failure of your regulation of appetite and satedness. This 'diabesity' is the consequence of the failure of this process. Moreover the spectrum of emergent pathologies will often differ between patients who have the same or similar medical condition. This illustrates some of the complex and fundamentally significant issues facing the medical researcher. The measurement of a single biomarker is an inherently flawed and limited concept i.e. the quality of the data is questionable! [3] There is a need to understand the mechanisms by which the brain regulates the body's function.

(Note: the author does not suggest that this process, of medical diagnosis, should be altered but instead that its limitations be understood. Such methodology is often irreplaceable as clinicians seek to sustain the lives of patients. Nevertheless there is an overwhelming need for new techniques, especially so in the primary care environment, which have the potential to reduce the cost and complexity of medicine.)

The factors which influence rate of reaction are typically $\mathrm{pH}$, catalysts, pressure, temperature, and pro-factors; yet in biology the fundamentals of chemistry have been overlooked or even abandoned. Similarly, in biology the understanding that there are physiological systems (e.g., pH, blood pressure, blood glucose, sleep, etc.) has been overlooked in the headlong rush to understand molecular biology. These are extraordinary examples of the way that healthcare, by accident or design, has incorporated a wide range of inherent limitations into the healthcare process.

The influence of acidity $(\mathrm{pH})$ is overlooked in the vast majority of clinical studies yet the reactivity of proteins is finely regulated by the prevailing $\mathrm{pH}$ which influences the prevailing levels of minerals [4]. If intercellular acidity increases the levels (or bioavailability) of calcium, magnesium, zinc and chromium will decline whilst the levels of other minerals - typically known as the transition or heavy metals - will increase e.g., iron, aluminium, mercury, cadmium, etc. If so, metabolic rate which requires essential minerals to catalyse the normal physiological biochemistries will function at a lower than normal rate whilst the pathological processes and/or 'oxidative stress', may become more prevalent.

In addition, the prevailing $\mathrm{pH}$ influences protein conformation i.e. whether a protein will adopt its coiled reactive state or its uncoiled unreactive state. This leads to problems of protein reactivity or where a particular protein will 'resist' its metabolic involvement e.g., insulin 'resistance', leptin 'resistance', etc. The phenomena of protein coiling has been researched but largely discarded. It is an inconvenient issue yet it is a significant issue in the etiology of many common pathologies.

To further complicate the issue, most proteins require to be raised to their activated state before they are able to react with their reactive state. To do so they require energy which can be provided by heat, vibration or light. Proteins start to denature at circa $40^{\circ} \mathrm{C}$ therefore this appears to discount the influence of heat. The effect of resonance or vibration - at least that which is provided in situ - would also therefore appear to be relatively insignificant. Most proteins are visually active therefore this appears to support the contention that light provides the energy which is necessary to raise a protein to its reactive state and to react with its reactive substrate. Moreover, following reaction, proteins and/or enzymes often release light of different colour and energy as they decay to their unreactive states therefore the characteristics and intensity of the light released is unique to each pathological reaction [5]. This release of biophotons is proportional to every pathological reaction. This release of light, of 'bioluminescence', has been widely noted e.g., in diabetes where changes of blue-yellow colour perception are associated with the onset and progression of diabetes however this phenomena is not solely associated with diabetes [6-8]. Changes of colour perception are a feature of almost every pathological process. It forms the basis of techniques which seek to photograph the 'aura' - because the body is highly bioluminescent - however the most significant manifestation of this phenomenon is that it influences colour perception and it is upon this phenomena that Strannik Virtual Scanning is based. Furthermore the technique is entirely safe and does not have any side-effects, or adverse or limiting factors.

\section{Strannik Technology}

Dr. Grakov, developer of the Strannik or Virtual Scanning technology, has been able to refine this technique - to develop the first mathematical model of the autonomic nervous system - to diagnose (i) the extent of genotype and phenotype for every common pathology at the level of molecular biology, (ii) the extent of associated cellular changes, (iii) to define in mathematical terms the level of organ instability and (iv) the level of systemic instability i.e. at the level of the physiological system(s) - including an understanding of the 'pathological functional system'; and (v) based upon the understanding that the brain continuously regulates the body's stability - by a frequency-related mechanism - as a therapeutic modality. It is a technique of apparently extraordinary precision and effectiveness.

It is increasingly recognised that genome sequence information is not sufficient i.e. that in order to maintain wellness it is necessary to understand the molecular mechanisms which result from altered genetic sequence (genotype) and, in particular, the prevailing lifestyle and/or environment (phenotype) which remains the single most important cause of morbidity and premature mortality. (Note: in this context the term 'phenotype' refers to the influence of lifestyle and the environment). Moreover many apparently healthy people may be suffering from early onset of pathologies which are manifest below the symptomatic level and do not yet influence their quality of life but which could do so in the future e.g., it is an inevitable fact that our ability to produce proteins declines as we age [9].

There is therefore a need to recognise that there are two elements to every pathology: genotype and phenotype [10]; but that stressful experience can cause physiological symptoms. Stress leads to the expression of phenotype and ultimately to genetic change. In simple terms (i) the rate at which our genes express a protein and (ii) the rate at which the expressed proteins react with their reactive substrate. It illustrates that genotype and phenotype are comorbidities and also, 
therefore, that in order to precisely define a condition e.g., diabetes (type 1 and type 2), it is necessary to define the extent of these two comorbidities. This is especially significant when considering whether a patient should be prescribed insulin, treatment involving drugs, whether they should be advised to adopt an exercise and dietary regime, or other modes of intervention.

Furthermore the rate of genetic expression is also subject to the laws of chemistry. In particular transferase enzymes often require magnesium or zinc to sustain their function and must therefore be influenced by acidity i.e. genetic expression of a protein is influenced by acidity in addition to the chemical and structural organisation of our DNA and genes. (Ultimately our predisposition to diabetes will be influenced by $\mathrm{pCO}_{2}$ in air, the levels of acidity which we ingest e.g., in drink, levels of fat, and our ability to exhale $\mathrm{CO}_{2}$ through exercise).

In addition there are three further issues which serve to complicate genetic data even more: (i) if someone becomes obese their genetic profile will alter yet if they adopt an exercise program and recover their original body weight their genetic profile will recover to its original state [11] and (ii) the genetic profile is context development i.e. different genetic profiles can express the same protein [12]. The genetic profile of the diabetic in many in SE India differs from that of the Caucasian. It is clear therefore that genetic change may in some cases be the cause of a particular problem but in other cases it may be the consequence of the problem; (iii) most genes do not act in isolation; (iv) the existence of a genetic defect does not necessarily indicate the onset or progression of a morbidity [13]; (v) the presence of disease-causing mutations in animals may have no significant effect upon the health of a human [14]. It is suggested that this is evidence of a neuro-regulatory mechanism. Nicholson ably summarises the issue when commenting upon 'the unexpected complexity of the human genome and the difficulties in accurately and unequivocally describing genotypes and phenotypes [15].

Accordingly, where is the evidence that frequency of different levels could have a physiological effect? Perhaps the simplest observation of this phenomena is in the area of migraine where flashing lights are known to induce photosensitive migraine yet flashing light therapy(s) have also been approved to treat photosensitive migraine, or that during sleep or a coma the dominant state adopted by the brain is the deltafrequency state. There is a scientific principle which has been recognised by biofeedback researchers although the fundamental principle remains beyond the current level of understanding. As a result the outcome of techniques such as colour therapy, biofeedback, neurofeedback, photic stimulation, biophotonics, and neuromodulation remains unreliable and consequently quite controversial.

This presents a problem for the biochemist. Perhaps Einstein aptly summarised the problem when he stated if we knew what we were doing it would not be called research'. How is it possible to justify that the complex biochemical entity that is the human body can be regulated by frequency yet their research is based upon providing biochemical input, usually via the digestive system, to alter the body's function and ameliorate the symptoms which are associated with a particular ailment. Perhaps the problem for biomedical researchers is the reverse question i.e. how can the complex range of chemistries explain the apparently coordinated function of the immensely regulated multisystemic entity that is the human body - bearing in mind that there are two entirely different but mutually dependent processes at play the simultaneous influence of sensory and biological data. Moreover what is the function of the neural networks? How do we explain their significance if not through regulation of a functional system?
It becomes increasingly evident that neural networks are often organised in neuronal networks which interact through a rhythmic frequency-based mechanism and, moreover, that these oscillatory interactions [16], which we observe as EEG frequencies, are observed across four main brain levels - known commonly as the beta, alpha, theta and delta ranges - and that they are associated with the integrated relationship involving sensory and cognitive function, brain function, neural networks, autonomic nervous system and the functional systems and pathological onset.

The issue was put into context by eminent geneticist Professor Eric Lander who led one of the teams to decipher the human genome and who identified that the human genome is the equivalent of a 'parts list' and that what was/is required is an 'operating manual to make it go'. As a result the world of biology is in disarray and denial yet there are many precedents which illustrate that the body responds to different electromagnetic frequencies. It becomes increasingly evident that the body's function responds to frequency at different levels of organisational and physiological significance i.e. at the neurological level in which the precise organisation of the neurons in neural networks regulates specific functional aspects of the body's function, at the level of the organ systems (physiological system) and individual organs, and at the cellular and molecular level. Accordingly the issue at stake is how to understand and adapt the phenomena.

There is one further aspect of the phenomena which has yet to be grasped i.e. that the brain regulates the function of the visceral organs. This illustrates that changes of cellular and molecular biology are often the consequence, not the cause, of the progressive failure of the brain to maintain autonomic stability. It means that biochemical changes e.g., as a result of drugs, nutrition; are largely treating the consequence of autonomic dysfunction - not the fundamental cause. This does not mean that drugs will be ineffective but it does mean that there may be a more effective way of treating the problem, perhaps in combination with drugs or perhaps as an alternative therapeutic modality, and that the effectiveness of drugs may erode as a result of the brain's neural regulation of visceral function.

\section{Strannik Virtual Scanning}

The Strannik Virtual Scanning technique uses measurements of colour perception as the data sets for a mathematical model of the autonomic nervous system [17-21]. It adopts a top-down philosophy i.e. from the brain to the viscera, by contrast with the vast bulk of medical research into the autonomic nervous system by contemporary biochemists who seek to develop and/or use drugs to modify autonomic dysfunction. The problem for this bottom-up philosophy is as follows: (i) that the brain is continuously adjusting the body's function to maintain or sustain its optimum stability, (ii) that autonomic instability is characterised by a spectrum of pathological indications - each comprising a genetic and phenotypic (environmental) component throughout all $30+$ organs and 13 physiological systems, (iii) the results of biomarker tests are inherently inaccurate i.e. the clinical context in which the test is used is limited or invalidated by the complex nature of the patient's health; (iv) that the autonomic nervous system, and the genotypic and phenotypic manifestations thereof, varies according to lifestyle and environmental factors which influence the patient; and (v) that neural plasticity and function is influenced by pathological onset and/or progression.

The results are presented in a context-specific manner which enables the GP to assess patient's health in different levels of physiological significance e.g., to identify (i) changes of psychological profile which 
Citation: Ewing GW (2015) Back to Basics: Limitations of Research Influencing the Human Brain Project. J Comput Sci Syst Biol 8:6 322-326. doi:10.4172/jcsb.1000206

are associated with the onset of pathology(s), (ii) pathological onset and/or progression at the molecular and cellular levels, (iii) the most dysfunctional systems and organs, (iv) whether 'pathological functional systems' have been established which accompany chronic onset of a particular medical condition. Initial indications, reported by respected academics and clinicians at various Russian medical institutes, are that SVS is $2-23 \%$ more accurate than the range of contemporary diagnostic tests against which it has been compared and which have been routinely used in these medical institutes.

The SVS technique is inclusive of all factors which influence rate of reaction and of pathological development. That the test includes measurements of colour perception is self-evident however it is also adjusted to include an assessment of how memory influences the patient's ability to undertake the test, the unique way which they do so, and inclusion of the patient's unique parameters i.e. age, weight and gender.

\section{Strannik Light Therapy}

Strannik Light Therapy (SLT) is based upon this principle i.e. that frequency can be adapted to regulate the body's function [22]. The SVS test identifies the unique patient profile and hence the precise parameters of the biofeedback program - which the patient installs on their PC and which provides the therapy which they undertake in their home.

SLT has been able to improve patient health in cases where the NHS has been completely unable to understand the nature of an ailment or to resolve the problem by current therapeutic techniques. That SLT has been able to resolve such problems - reported by Russian clinicians to be typically $83-96 \%$ effectiveness - is proof of a truly remarkable level of understanding of how the brain regulates the body's function.

\section{The Significance of Strannik Technology viz a viz the Human Brain Project}

Such a technology is validated by the mere existence of the Human Brain Project which incorporates a number of key aims and objectives including the following issues which are currently covered by Strannik technology $[2,23,24]$ and/or where the use of Strannik technology has the potential to make a significant contribution to HBP research (in particular (ii)-(iv), (vi)) in adjacent areas of research:

(i) To understand the processes which enable our brain to function,

(ii) identify specific brain regions or neural assemblies which are involved in specific tasks;

(iii) The likely factors and/or circuitry which connects these brain regions or neural assemblies,

(iv) Establish the principles of information processing within and between these brain regions;

(v) Develop mathematical models of brain function, (vi) provide models of synaptic plasticity;

(vii) Provide models of cognitive functions including attention, working memory, and sleep;

(viii) Identify general principles of neural computation and the design and implementation of Neuromorphic Computing Systems;

(ix) Develop multi-scale, multi-level (genes to whole brain), models of the human brain in which different brain areas are modelled at levels of detail appropriate to the current knowledge and data;

(x) Develop new comprehensive classification of diseases based upon combinations of identified pathologies and markers - which would lead to the development of a new category of biologically-based diagnostics, supported by strong mechanistic hypotheses of disease causation;

(xi) Characterise the psychometric function calculated by the brain model;

(xii) Identify differential disease signatures from clinical data and to develop new nosological classifications based upon biological dysfunction rather than symptoms and syndromes [18,25-29];

(xiii) Use disease models to identify novel treatment strategies [21,30-32], and (xiv) develop treatment strategies for personalised medicine which have been adapted for a specific condition or patient.

\section{References}

1. Rethinking the Brain (2015) Critics of the European Human Brain Project were justified, says an independent report on the project. Both its governance and its scientific direction need to be adjusted. Nature 519: 389 .

2. Ewing GW, Grakov IG (2015) A Comparison of the Aims and Objectives of the Human Brain Project with Grakov's Mathematical Model of the Autonomic Nervous System (Strannik Technology). Enliven: Neurol Neurotech 1: 002.

3. Jacqui Wise (2008) NHS must make greater use of information technology. BMJ 337: a2303.

4. Ewing GW (2012) pH is a Neurally Regulated Physiological System. Increased Acidity Alters Protein Conformation and Cell Morphology and is a Significant Factor in the Onset of Diabetes and Other Common Pathologies. The Open Systems Biology Journal 5: 1-12.

5. Ewing GW, Parvez SH, Grakov IG (2011) Further Observations on Visual Perception: the influence of pathologies upon the absorption of light and emission of bioluminescence. The Open Systems Biology Journal 4: 1-7.

6. Daley ML, Watzke RC, Riddle MC (1987) Early loss of blue-sensitive color vision in patients with type I diabetes. Diabetes Care 10: 777-781.

7. Kurtenbach A, Schiefer U, Neu A, Zrenner E (1999) Preretinopic changes in the colour vision of juvenile diabetics. $\mathrm{Br} \mathrm{J}$ Ophthalmol 83: 43-46.

8. Beisswenger PJ, Makita Z, Curphey TJ, Moore LL, Jean S, et al. (1995) Formation of immunochemical advanced glycosylation end products precedes and correlates with early manifestations of renal and retinal disease in diabetes. Diabetes 44: 824-829.

9. Johnston JJ, Lewis KL, Ng D, Singh LN, Wynter J, et al. (2015) Individualized iterative phenotyping for genome-wide analysis of loss-of-function mutations. Am J Hum Genet 96: 913-925.

10. Ewing GW, Parvez SH (2010) The multi-systemic nature of diabetes mellitus: Genotype or phenotype? N Am J Med Sci 2: 444-456.

11. Booth FW, Chakravarthy M, Spangenburg EE (2002) Exercise and gene expression: physiological regulation of the human genome through physical activity. J Physiol 543: 399-411.

12. Bodhini D, Radha V, Ghosh S, Majumder PP, Mohan V (2011) Lack of association of PTPN1 gene polymorphisms with type 2 diabetes in south Indians. J Genet 90: 323-326.

13. Jordan DM, Frangakis SG, Golzio C, Cassa CA, Kurtzberg J, et al. (2015) Identification of cis-suppression of human disease mutations by comparative genomics. Nature 524: 225-229.

14. Vu V, Verster AJ, Schertzberg M, Chuluunbaatar T, Spensley M, et al. (2015) Natural Variation in Gene Expression Modulates the Severity of Mutant Phenotypes. Cell 162: 391-402.

15. Nicholson JK (2006) Global systems biology, personalized medicine and molecular epidemiology. Mol Syst Biol 2: 52

16. Bosman CA, Aboitiz F (2015) Functional constraints in the evolution of brain circuits. Front Neurosci 9: 303. 
Citation: Ewing GW (2015) Back to Basics: Limitations of Research Influencing the Human Brain Project. J Comput Sci Syst Biol 8:6 322-326. doi:10.4172/jcsb.1000206

17. Grakov IG. Strannik Diagnostic and Treatment System: a Virtual Scanner for the Health Service. Minutes of Meeting No. 11 of the Praesidium of the Siberian of the Academy of Medical Sciences of the USSR (AMN) held in Novosibirsk 4 December 1985.

18. Ewing GW, Ewing EN (2008) Virtual Scanning - a new generation of medical technology - beyond biomedicine? Pub Montague Healthcare Books.

19. Hankey A, Ewing E (2007) New light on chromotherapy: Grakov's 'Virtual Scanning' system of medical assessment and treatment. Evid Based Complement Alternat Med 4: 139-144.

20. Ewing G, Ewing E, Hankey A (2007) Virtual scanning-a new system of medical assessment and treatment: Part I. Assessment. J Altern Complement Med 13: 271-285

21. Vysochin Yu (2001) Methodology and Technology of Invigoration of Different Population Orders. In: Consolidated 5 year Research Plan of Physical Training, Sports and Tourism State Committee of the Russian Federation.

22. Ewing GW (2009)A Theoretical Framework for Photosensitivity: Evidence of Systemic Regulation. Journal of Computer Science and System Biology 2: 287-297.

23. www.montaguehealthcare.co.uk/presentation.php

24. www.montaguehealthcare.co.uk/OperatingManual.pdf

25. Ewing GW, Ewing EN, Parvez SH (2009) The Multi-systemic Origins of Migraine. Biogenic Amines 23: 1-52.
26. Ewing GW, Parvez SH (2012) The Influence of Pathologies upon Sensory Perception and Sensory Coordination in Children with Developmental Dyslexia and Learning Disorders: A Unified Theory of Developmental Dyslexia. N Am J Med Sci 4: 109-116.

27. Ewing GW, Ewing EN, Parvez SH (2009) Developmental Dyslexia: the link with the Autonomic Nervous System and the Physiological Systems. J Biogenic Amines 23: 115-190.

28. Ewing GW (2015) Case Study: The Determination a Complex Multi-Systemic Medical Condition by a Cognitive, Virtual Scanning Technique. Case Reports in Clinical Medicine 4: 209-221.

29. Nwose EU, Ewing GW (2009) Computer diagnosis in cardiology: oxidative stress hypothesis. N Am J Med Sci 1: 220-225.

30. Nwose EU, Ewing GW, Ewing EN (2009) Migraine can be managed with Virtua Scanning: Case report. The Open Complementary Medicine Journal 1: 16-18.

31. Ewing GW, Nwose EU, Ewing EN (2009) Obstructive sleep apnea management with interactive computer technology and nutrition: two case reports. J Altern Complement Med 15: 1379-1381.

32. Ewing GW (2015) The successful treatment of Dysarthria using Strannik Light Therapy: a case study. Case Reports in Clinical Medicine 4: 266-269. 